\title{
Why do Chaotic Orbits Converge under a Random Velocity Reset?
}

\author{
Yih-Yuh Chen \\ Physics Department, National Taiwan University, Taipei, Taiwan, Republic of China
}

(Received 8 July 1996)

\begin{abstract}
When an ensemble of interacting identical particles is put inside a confining potential it is usually the case that the particle trajectories are chaotic and thus exhibit sensitive dependence on initial conditions. However, if their velocities are randomly reset at a regular time interval $\tau$ according to a prescribed probability distribution, then it might happen that all the particles will eventually converge to some unique yet irratic orbit in the physical space, depending on the pertinent physical parameters of the system and $\tau$. We suggest that this is a rather general phenomenon, and give an argument in favor of this view. Scaling properties for the threshold reset time are also derived. [S0031-9007(96)01681-X]
\end{abstract}

PACS numbers: 05.45.+b

In many applications one usually is concerned about how to impose a certain behavior on a dynamical system using some conveniently chosen driving mechanism. At the heart of this type of control is that the dynamical response of the system under consideration be stable with respect to the driving reset signal, for otherwise a runaway situation will ensue. The study of this type of stability for a physical system is quite interesting when the system is already in a chaotic state, and even more so when the resetting signal itself is also random or chaotic, because there is no telling how compounded seemingly random events will exhibit themselves. In this sense, we can say that the work of Pecora and Carroll [1] and that of Fahy and Hamann [2] represent one intriguing feature of a chaotic system, namely, we can maintain stability on an otherwise chaotic system using either a chaotic or a random resetting signal.

To see this similarity more clearly, we recall that in the original implementation of the synchronization of chaos [1] one simply channels one output of a chaotic driving system to replace the corresponding variable of an identical response system, and observes that the other variables of the response system evolve into the same state as the driver, irrespective of the initial conditions. On the other hand, for the Fahy and Hamann implementation $[2,3]$, one starts with two noninteracting particles moving chaotically inside a confining potential. The velocities of both particles are then randomly reset according to the Maxwellian distribution at a regular time interval $\tau$, and it is observed that for $\tau$ less than a threshold value the trajectories always tend to converge to the same orbit, independent of their initial conditions. In these two examples, the only difference in their resetting mechanism is that in the former the reset is done continuously in time, whereas in the latter it is done at a discrete time interval. This suggests two possible immediate generalizations of the cited work: (1) We can investigate synchronous chaos within the Pecora-Carroll implementation using a discrete time reset, and (2) we can study the generality of the Fahy-Hamann result. Because some interesting progress and potential applications have been reported [47] concerning possibility (1), here we will concentrate on (2) only.

To set the stage, we assume that an ensemble of interacting identical particles is put inside a confining force field so that total potential energy is $V(\vec{x})$ when the configuration space coordinate of the system is $\vec{x}$. (For an $n$-body system moving in a $D$-dimensional space, $\vec{x}$ has $N \equiv n D$ components.) For simplicity, we assume the potential $V(\vec{x})$ is a smooth function which never blows up unless $\|\vec{x}\| \rightarrow \infty$. The equation of motion for the system then reads

$$
\frac{d \vec{x}}{d t}=\vec{v} \quad \text { and } \quad \frac{d \vec{v}}{d t}=-\frac{1}{m} \frac{\partial V}{\partial \vec{x}}-\gamma \vec{v},
$$

where $m$ is the mass of the particles, $\vec{v}$ the velocity conjugate to $\vec{x}$, and we have included a damping factor $\gamma \geq 0$ to take into account a possible dissipative effect. The system will freely evolve for a time $\tau$ before suffering an instantaneous reset in the velocity $\vec{v}$ from an external agent. The new velocity $\vec{v}_{\text {new }}$ after reset takes the following form:

$$
\vec{v}_{\text {new }}=\alpha \vec{v}_{\text {old }}+\vec{v}_{\text {random }},
$$

where the parameter $\alpha$ is included to simulate a certain memory effect, and $\vec{v}_{\text {random }}$ is a velocity randomly selected from a prescribed distribution. In the following discussion we shall confine ourselves to the case $0 \leq \alpha \leq 1$ $[2,3]$. Disregarding the possible gain from the random reset, the system tends to lose a fraction of its kinetic energy after each reset when $\alpha<1$. We thus expect $\alpha$ and $\gamma(\geq 0)$ to play a similar role in the formalism.

In order to study the linear stability of the system, we will have to calculate the time evolution of the small deviation between two nearby orbits in the phase space and see if it converges exponentially. The deviations $\delta \vec{x}$ and $\delta \vec{v}$ satisfy

$$
\frac{d \delta \vec{x}}{d t}=\delta \vec{v} \quad \text { and } \quad \frac{d \delta \vec{v}}{d t}=-\frac{1}{m} \frac{\partial^{2} V}{\partial \vec{x}^{2}} \delta \vec{x}-\gamma \delta \vec{v}
$$

Suppose the system is allowed to evolve for a short time $\tau$, then we can integrate Eq. (3) forward in time and express the answer in a Taylor series of $\tau$. Correct to 
second order in $\tau$, one has

$$
\begin{aligned}
& \delta \vec{x}=\delta \vec{x}_{0}+\delta \vec{v}_{0} \tau-\frac{\tau^{2}}{2 m}\left(\frac{\partial^{2} V_{0}}{\partial \vec{x}^{2}} \delta \vec{x}_{0}+m \gamma \delta \overrightarrow{\boldsymbol{v}}_{0}\right)+\cdots, \\
& \delta \vec{v}=\delta \overrightarrow{\boldsymbol{v}}_{0}-\frac{\tau}{m}\left(\frac{\partial^{2} V_{0}}{\partial \vec{v}^{2}} \delta \vec{x}_{0}+m \gamma \delta \overrightarrow{\boldsymbol{v}}_{0}\right)-\frac{\tau^{2}}{2 m}\left(\frac{\partial^{3} V_{0}}{\partial \vec{x}^{3}} \overrightarrow{\boldsymbol{v}}_{0} \delta \vec{x}_{0}+\frac{\partial^{2} V_{0}}{\partial \vec{x}^{2}} \delta \overrightarrow{\boldsymbol{v}}_{0}-\gamma \frac{\partial^{2} V_{0}}{\partial \vec{x}^{2}} \delta \vec{x}_{0}-m \gamma^{2} \delta \vec{v}_{0}\right)+\cdots,
\end{aligned}
$$

where the subscript " 0 " means that a quantity is evaluated at the reference time. After this brief free evolving moment, the system is subjected to a reset described by Eq. (2). But since the two nearby orbits suffer the same amount of random reset from the term $\vec{v}_{\text {random }}$, the $\delta \vec{v}$ after the reset is necessarily $\alpha$ times that computed in Eq. (4). Therefore, the (linear) relation between $\left(\delta \vec{x}_{0}, \delta \vec{v}_{0}\right)$ of the previous reset and $(\delta \vec{x}, \delta \vec{v})$ after the new reset can be succinctly organized in the matrix form $(\delta \vec{x}, \delta \vec{v})^{T}=H\left(\delta \vec{x}_{0}, \delta \vec{v}_{0}\right)^{T}$, with

$$
\begin{aligned}
H \equiv & \left(\begin{array}{cc}
I & 0 \\
0 & \alpha I
\end{array}\right)+\tau\left(\begin{array}{cc}
0 & I \\
-\frac{\alpha}{m} \frac{\partial^{2} V_{0}}{\partial \vec{x}^{2}} & -\alpha \gamma I
\end{array}\right) \\
& -\frac{\tau^{2}}{2 m}\left(\begin{array}{cc}
\frac{\partial^{2} V_{0}}{\partial \vec{x}^{2}} & m \gamma \\
\alpha \frac{\partial^{3} V_{0}}{\partial \vec{x}^{3}} \overrightarrow{\boldsymbol{v}}_{0}-\alpha \gamma \frac{\partial^{2} V_{0}}{\partial \vec{x}^{2}} & \alpha \frac{\partial^{2} V_{0}}{\partial \vec{x}^{2}}-\alpha m \gamma^{2}
\end{array}\right),
\end{aligned}
$$

where $I$ is an $N \times N$ identity matrix. The investigation of the stability of the system then reduces to solving for the compounded effect of the matrix $H$ at different times and verifying that it does not blow up in the long run. But since this obviously presents a tremendous challenge for analytical treatment, we will resort to a less direct but more intuitive approach: trying to evaluate the average of $H$ and obtaining its stability criterion, which, in this case, is simply that the magnitude of the eigenvalues of the averaged $H$ are less than unity.

But before we proceed, a few remarks are in order. First, in view of the fact that the system is roaming erratically in the phase space, it seems reasonable to assume that after a long time there will be a steady probability distribution $f(\vec{x}, \vec{v})$ for the system to be found at the state $(\vec{x}, \vec{v})$. By our very construction, the isotropic velocity distribution is independent of the position distribution after each reset, implying that $f(\vec{x}, \vec{v})$ can be factored into $f_{1}(\vec{x}) \cdot f_{2}(\|\vec{v}\|)$ for some functions $f_{1}$ and $f_{2}$. Borrowing an idea from statistical mechanics, we will further make the (as yet unsubstantiated) assumption that $f_{1}(\vec{x})=g(V(\vec{x}))$ for some monotonically decreasing function $g(V)$ which vanishes quickly as $V$ tends to infinity.

To evaluate the average value of the various matrix elements, we first note that the average of $\frac{\partial^{3} V_{0}}{\partial \vec{x}^{3}} \overrightarrow{\boldsymbol{v}}_{0}$ vanishes due to symmetry in $f_{2}\left(\left\|\vec{v}_{0}\right\|\right)$. Then all that remains to evaluate is

$$
\begin{aligned}
L & \equiv \frac{1}{m}\left\langle\frac{\partial^{2} V_{0}}{\partial \vec{x}^{2}}\right\rangle=\frac{1}{m} \int g(V) \frac{\partial^{2} V}{\partial \vec{x}^{2}} d \vec{x} \\
& =-\frac{1}{m} \int \frac{d g}{d V} \frac{\partial V}{\partial \vec{x}} \frac{\partial V}{\partial \vec{x}} d \vec{x}
\end{aligned}
$$

after integration by parts. (The angular brackets stand for the phase space average of a quantity.) The potential energy resulting from the pairwise mutual interactions between the particles has a reflection symmetry under $\vec{x} \rightarrow-\vec{x}$. Hence the tensor $L$ is a positive-definite operator if the external potential also has a reflection symmetry, because then the cross terms $\frac{\partial V}{\partial x^{i}} \frac{\partial V}{\partial x^{j}}(i \neq j)$ will cancel out, leaving only the diagonal elements, and because $d g / d V$ is negative by assumption. We therefore further restrict ourselves to the case when the external confining field possesses a reflection symmetry from now on. This much said, now we can proceed with the analysis of the eigenvalues of $H$.

Because the matrix elements of $H$ now involve only the two commuting operators $I$ and $L$, solving the eigenvalue problem $H \psi=\lambda \psi$ becomes quite trivial. In fact, denoting the eigenvalue of $L$ by $l$, one can easily derive

$$
\begin{aligned}
\lambda= & \frac{1}{2}\left[1+\alpha-\alpha \gamma \tau-\frac{\left(l+\alpha l-\alpha \gamma^{2}\right)}{2} \tau^{2} \pm A^{1 / 2}\right], \\
A \equiv & (1-\alpha)^{2}+2 \alpha(1-\alpha) \gamma \tau-\left[(1+\alpha)^{2} l-\alpha(2 \alpha-1) \gamma^{2}\right] \tau^{2}+\left(3 l+\alpha l-\alpha \gamma^{2}\right) \alpha \gamma \tau^{3} \\
& +\left[\left(l+\alpha l-\alpha \gamma^{2}\right)^{2} / 4-\alpha l^{2}\right] \tau^{4} .
\end{aligned}
$$

If $\alpha<1$, then for all small enough $\tau$ we may further expand Eq. (7) in powers of $\tau$. This yields

$$
\lambda \approx 1-\frac{1}{2} \frac{1+\alpha}{1-\alpha} l \tau^{2}, \quad \alpha(1-\gamma \tau) \text { if } \gamma>0,
$$

and

$$
\begin{gathered}
\lambda \approx 1-\frac{1}{2} \frac{1+\alpha}{1-\alpha} l \tau^{2}, \\
\alpha\left(1+\frac{1}{2} \frac{1+\alpha}{1-\alpha} l \tau^{2}\right) \quad \text { if } \gamma=0 .
\end{gathered}
$$

Therefore the system is stable for all small enough $\tau$, provided that $\alpha<1$. Similarly, Eq. (7) predicts that for the case $\alpha>1$ the system is unstable if $\gamma=0$, no matter 
how small $\tau$ is. In this sense we see that the dissipative nature of $\alpha<1$ and $\gamma>0$ has a stabilizing effect on the system, just as one has expected.

At this point our analysis suggests only that the configuration of the system will converge to a unique orbit in the phase space, and not that every single particle in the system will eventually converge onto a unique trajectory in the physical space. However, because the particles are identical, we know that if $(\vec{x}(t), \vec{v}(t))$ is an admissible solution to the equation of motion, then the vector $\left(\vec{x}^{\prime}(t), \vec{v}^{\prime}(t)\right)$ obtained by exchanging only one pair of the particle indices of $(\vec{x}(t), \vec{v}(t))$ must also be a solution. In other words, we may view $(\vec{x}(t), \vec{v}(t))$ and $\left(\vec{x}^{\prime}(t), \vec{v}^{\prime}(t)\right)$ as two admissible evolving configurations in the phase space. But if this is true, then it means their difference $(\delta \vec{x}(t), \delta \vec{v}(t))$ necessarily obeys Eq. (3); and our previous analysis goes through. In effect, this then implies that if at any moment two particles chance into each other's neighborhood, they will eventually follow the same path whenever the stability criterion we derived above is satisfied. This is demonstrated in Fig. 1 for three particles of mass $m=20$ moving in the confining potential [2]

$$
V(x, y)=\sin (2 \pi x) / 2 \pi x+\sin (2 \pi y) / 2 \pi y+r^{s} / 16 \pi,
$$

with $s=4$ and under the influence of a mutual repulsive potential $V_{\text {rep }}\left(\left\|\vec{x}_{i}-\vec{x}_{j}\right\|\right)=50 /\left(\left\|\vec{x}_{i}-\vec{x}_{j}\right\|^{2}+1\right)$ between the particles when the reset time $\tau$ is 0.1 . Here $\alpha=\gamma=0$, and the random velocity $\vec{v}_{\text {random }}$ has an average magnitude of 250 .

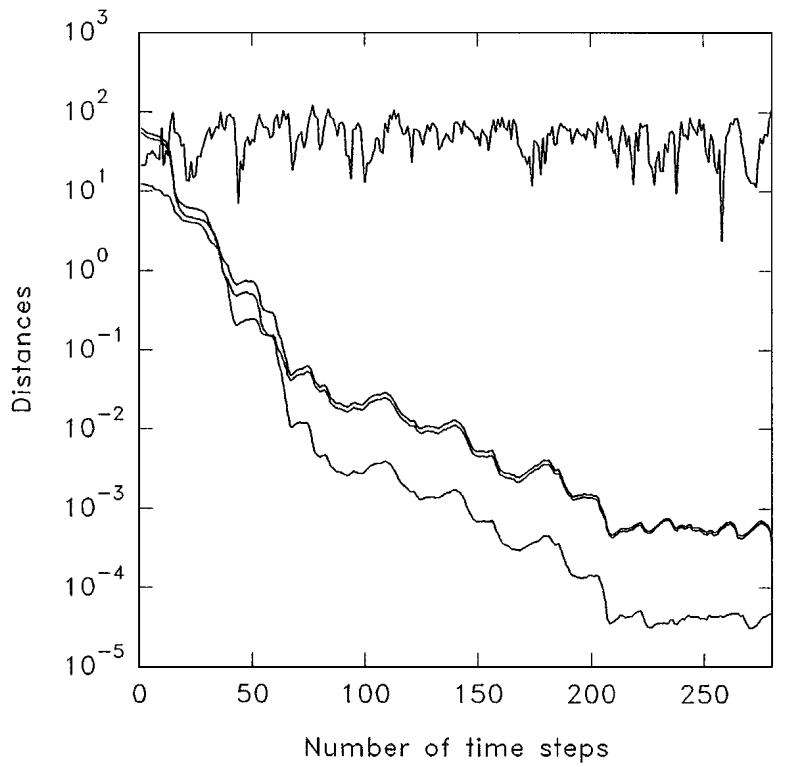

FIG. 1. Convergence of orbits for three mutually repulsive particles put inside a confining potential. The high lying curve is the distance of one reference particle to the origin; the remaining three (converging) curves are the relative distances of the particles.
The fact that the random reset velocity does not explicitly come into sight in the previous analysis also suggests that the detailed form of the distribution law for the random reset velocity should be inessential. This we have verified for at least two cases: the Maxwellian distribution and the uniform distribution with a cutoff. On the other hand, a statement of this generality puts us directly in a paradoxical situation, because by "toning down" the random velocity, i.e., setting $\vec{v}_{\text {random }}$ to infinity, one would predict exactly the same synchronous behavior, which looks ridiculous due to the fact that a system satisfying the stability condition and subject to no random velocity reset will keep losing the kinetic energy either continuously via the damping $\gamma$ (if $\gamma>0$ ) or discretely at each reset via $\alpha$ (if $\alpha<1$ ) until it comes to a complete stop when the potential energy achieves its local minimum. Yet there is no guarantee that particles arranged in a configuration of minimum potential energy will collapse into the same spatial point - they do not in general; and this is especially true if their mutual interaction is repulsive. So what is happening here?

The answer to this difficulty seems to lie in the time scales involved: When the random reset is weaker in magnitude, it will take a considerably longer time for the system to experience many potential lows and highs before the phase space averaging can make any sense. Besides, the same long waiting time is needed for the random reset to drive the system to a configuration that is favorable for particle collapse if the external potential is incapable of counteracting the repulsion between the particles initially. However, a quantitative analysis of this idea still needs to be worked out.

Because too long a waiting time $\tau$ is like not perturbing the originally chaotic system at all, we also expect there to be a threshold value $\tau_{c}$ above which orbital convergence is impossible. But how does $\tau_{c}$ depend on the system parameters? Indeed, our analysis also provides a hint to this question; and to simplify the matters we will treat the case $\gamma=0$ in the following as an illustration.

Clearly, convergence of orbits is possible only if the eigenvalue $\lambda$ of Eq. (7) has a norm less than unity. Although we cannot directly set the norm of Eq. (7) to unity to compute $\tau_{c}$ (because this equation is derived with the understanding that $\tau$ is small, an assumption which is rendered invalid when $\tau$ gets to as large as $\tau_{c}$ ), at least we are sure that the dimensionless parameter $l \tau_{c}^{2}$ must have increased to order one when instability sets in. Therefore, $\tau_{c}$ should obey the scaling law

$$
\tau_{c} \propto l^{-1 / 2},
$$

where a prefactor which might depend on $\alpha$ in some complicated way has been dropped because our simple theory is incapable of determining its precise form. But by Eq. (6) we see that $l$ may be estimated using $l \approx$ $\langle V\rangle / m\left\langle\vec{x}^{2}\right\rangle$. Hence Eq. (12) becomes $\tau_{c} \propto \sqrt{m\left\langle\vec{x}^{2}\right\rangle /\langle V\rangle}$.

To check the validity of this prediction, we may consider specific examples for $V$. For instance, if we 

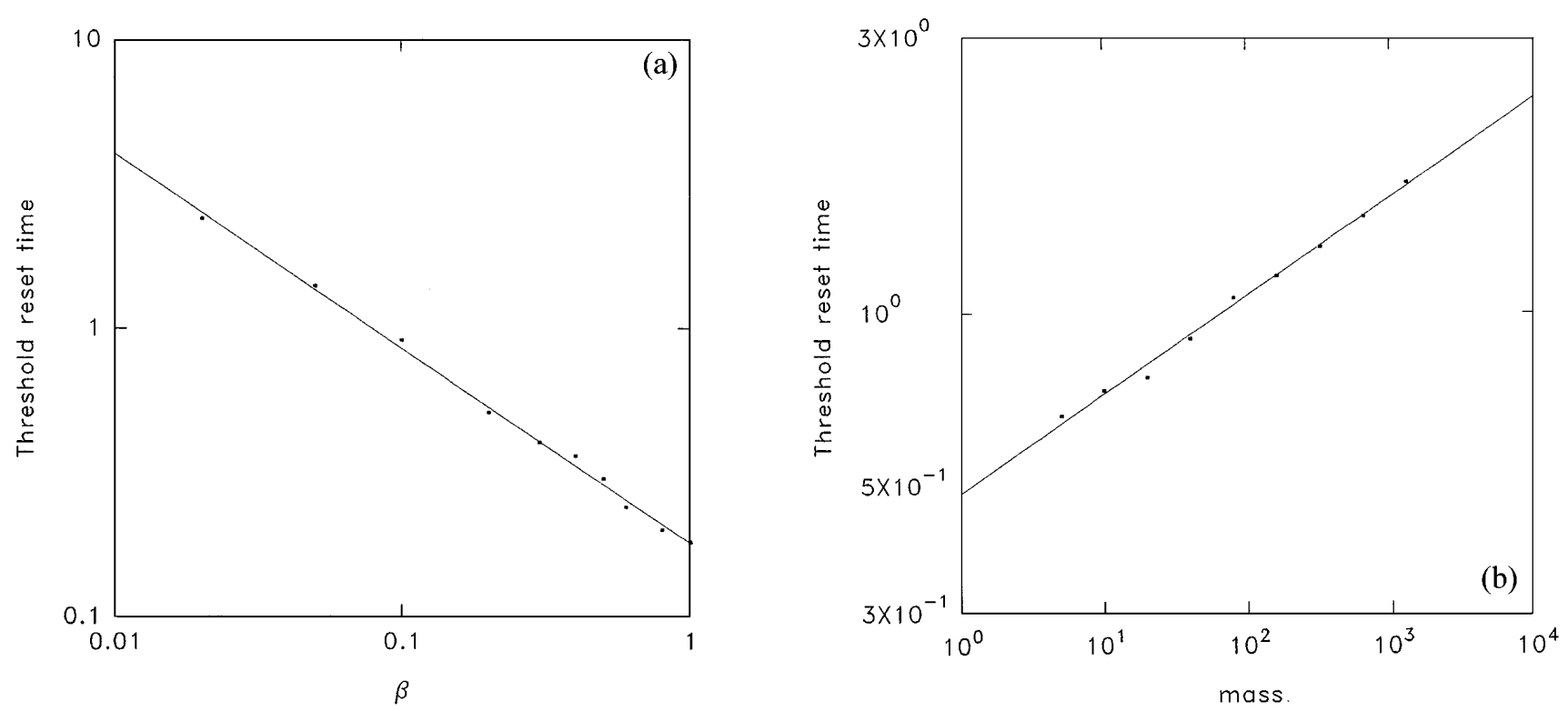

FIG. 2. The scaling behavior of the threshold reset time $\tau_{c}$ on (a) $\beta$, the strength of velocity reset when the particle mass $m$ is 20 , and on (b) the mass $m$ of the particle when $\beta=0.1$. (In both cases the average random reset velocity is 250 .) The numerical values of the slope in (a) and (b) are -0.68 and 0.17 , respectively, comparing favorably with the theoretical value of $-2 / 3$ and $1 / 6$.

have $V(\vec{x}) \propto \vec{x}^{s}$ as $\|\vec{x}\|$ tends to infinity, then $\tau_{c} \propto$ $m^{1 / 2}\langle V\rangle^{-\frac{1}{2}+\frac{1}{s}}$ when the average potential energy of the system is large. Since we also have $\langle V\rangle \approx \frac{1}{2} m \vec{v}_{\text {random }}^{2}$, the answer becomes $\tau_{c} \propto m^{1 / s}\left(\vec{v}_{\text {random }}^{2}\right)^{-\frac{1}{2}+\frac{1}{s}}$. Therefore, if we somehow normalize a velocity distribution $\vec{u}_{\text {random }}$ such that $\left\langle\left\|\vec{u}_{\text {random }}\right\|\right\rangle$ is a fixed number and rewrite the reset velocity as

$$
\vec{v}_{\text {new }}=\alpha \vec{v}_{\text {old }}+\vec{v}_{\text {random }} \equiv \alpha \vec{v}_{\text {old }}+\beta \vec{u}_{\text {random }}
$$

so that the parameter $\beta$ signifies the strength of the velocity reset from an external agent, then the prediction is

$$
\tau_{c} \propto m^{1 / s} \beta^{-1+\frac{2}{s}},
$$

which we have verified for the potential of Eq. (11) with $s=4$ and $s=6$, respectively. The latter is shown in Fig. 2.

In summary, generalizing previous works $[2,3,8]$ in several respects, we have shown that randomly resetting the particle velocity at a suitably chosen discrete time interval can bring the individual particle trajectories into convergence, even if there is a repulsive force between any two particles. And for this to occur, the detailed form of the velocity distribution is not essential, either. The threshold reset time $\tau_{c}$ depends on system properties such as the particle mass, the amplitude of the velocity reset, and the form of the potential energy. Under appropriate limits, we have also derived its dependence on these parameters. Despite all its apparent success, we should also keep in mind that our argument still lacks the kind of sophistication a complete theory should have. For one thing, we are unable to assess the validity of substituting the actual evaluation of the stability matrix by its phase space average; and the various assumptions explicitly or implicitly woven into the formalism are subject to open criticism and further investigation. We hope issues like these will be clarified in the future.

This work was supported by the National Science Council of the Republic of China under Grant No. NSC85-2112-M002-010 and the Gou-Pee Papers Foundation.

[1] L. M. Pecora and T. L. Carroll, Phys. Rev. Lett. 64, 821824 (1990).

[2] S. Fahy and D. R. Hamann, Phys. Rev. Lett. 69, 761-764 (1992).

[3] B. Kaulakys and G. Vektaris, Phy. Rev. E 52, 2091-2094 (1995).

[4] R. E. Amritkar and N. Gupte, Phys. Rev. E 47, 3889-3895 (1993).

[5] Y.-Y. Chen, "A Wait-and-Reset Strategy to Synchronizing Chaotic Systems" (to be published).

[6] Y.-Y. Chen, "Randomly synchronizing chaotic systems" (to be published).

[7] Y.-Y. Chen, Europhys. Lett. 34, 245-250 (1996).

[8] A. Maritan and J. R. Banavar, Phys. Rev. Lett. 72, $1451-$ 1454 (1994). Also see A. S. Pikovsky, Phys. Rev. Lett. 73, 2931 (1994); A. Maritan and J. R. Banavar, Phys. Rev. Lett. 73, 2932 (1994). 